\title{
Explorando a relação entre consciência morfológica, processamento cognitivo e escrita ${ }^{1}$
}

\author{
Exploring the relationship between morphological \\ awareness, cognitive processing and spelling
}

\author{
Márcia Maria Peruzzi Elia da MOTA²
}

\section{Resumo}

Estudos realizados em línguas alfabéticas mostram que a habilidade de refletir sobre os morfemas que compõem as palavras - consciência morfológica - está associada ao sucesso no reconhecimento e compreensão de palavras na leitura. O português é uma língua com uma ortografia bastante regular em termos das correspondências entre letra e som. Neste estudo, investiga-se se o processamento da morfologia contribui para a escrita no português e se essa contribuição é independente de outros aspectos cognitivos da linguagem, como vocabulário e memória auditiva. Crianças de $2^{\circ}$ ano e $3^{\circ}$ ano do Ensino Fundamental constituíram a amostra. Os resultados das correlações de Pearson mostram que a consciência morfológica está associada à escrita; quando se controla a memória e o vocabulário, essa relação passa a ser significativa.

Unitermos: Alfabetização. Cognição. Consciência metalinguística. Escrita.

\begin{abstract}
Studies carried out in alphabetic languages show that the ability to reflect upon morphemes, morphological awareness, is related to the success in recognizing words and comprehension in reading and spelling. Portuguese is a language with a fairy normal orthography in terms ofletter-sound correspondence rules. This study investigated whether morphological awareness helps with spelling in Portuguese and whether this contribution is independent of other cognitive aspects of language such as vocabulary and memory. The sample was composed of children from the $2^{\text {nd }}$ and $3^{\text {rd }}$ grades. The Pearson correlation results showed that morphological awareness is associated with spelling; when memory and vocabulary are controlled this relationship is significant.
\end{abstract}

Uniterms: Literacy. Cognition. Metalinguistic awareness. Spelling.

Morfemas são as menores unidades linguísticas que têm significado próprio. Joanne Carlisle, investigando crianças falantes do inglês, mostrou que a habilidade de refletir sobre os morfemas das palavras está associada ao desempenho na leitura de palavras isola- das (reconhecimento de palavras), à compreensão de leitura (Carlisle, 1995, 2000; Carlisle \& Fleming, 2003) e também ao desempenho da escrita (Carlisle, 1988; 1996). A habilidade de refletir sobre essas unidades de significado é chamada consciência morfológica e pode

\section{$\boldsymbol{U V \boldsymbol { V }}$}

1 Apoio: Fundação de Apoio à Pesquisa do Estado de Minas Gerais (Fapemig-APQ-processo nº 00679-8)

2 Universidade do Estado do Rio de Janeiro, Instituto de Psicologia. Campus Francisco Negrão de Lima, Pavilhão João Lyra Filho, R. São Francisco Xavier, 524 , $10^{\circ}$ andar, Bloco B, Sala 10019, Maracanã, 20550-900, Rio de Janeiro, RJ, Brasil. E-mail: <mmotapsi@gmail.com>.

Agradecimentos: Aos bolsistas de Iniciação Científica do Conselho Nacional de Desenvolvimento Científico e Tecnológico: Stella Mansur-Lisboa e a bolsistas da Fapemig Jaqueline Dias. 
ajudar o aprendiz a ler e a escrever (Carlisle, 1995), uma vez que a ortografia de muitas palavras depende da sua origem. Palavras como "açucarado", que têm ortografia ambígua, podem ser escritas de forma correta quando se sabe sua origem:"açúcar". Nos últimos anos, verificou-se um crescimento dos estudos que investigam o papel da consciência morfológica na leitura e na escrita. Gradualmente, esses estudos estão delineando como essa habilidade se desenvolve e como se relaciona com a leitura e a escrita (Duncan, Casalis \& Cole, 2009).

Outras pesquisas realizadas com falantes do inglês obtiveram resultados semelhantes aos estudos já citados, tanto no âmbito da leitura (Deacon \& Kirby, 2004; Nagy, Berninger \& Abbot, 2006), como também nas investigações sobre a escrita (Deacon \& Bryant, 2005; Nunes, Bindman \& Bryant, 1997). O inglês é uma língua com uma ortografia mais "opaca" - por ter muitas palavras irregulares - do que o português. Palavras irregulares são palavras que não obedecem a regras de correspondência entre letra e som. Muitas irregularidades no inglês podem ser atribuídas à manutenção da estrutura morfológica das palavras. Por exemplo, palavras como heal e health são escritas da mesma forma, porque mantém uma relação semântica, embora sejam pronunciadas de maneiras diferentes,

Mann (2000) argumenta que, quando as palavras podem ser escritas ou lidas por correspondência letra e som, o processamento morfológico pode ter um peso menor do que o processamento fonológico. Entretanto, autores como Lehtonen e Bryant (2005) afirmam que a hipótese de que a consciência morfológica contribui para leitura e escrita em línguas cuja ortografia obedece às regras de correspondência letra e som é pertinente e precisa ser investigada empiricamente.

Assim, há palavras morfologicamente complexas que não são ambíguas e podem ser escritas pela aplicação das regras de correspondência entre letra e som. É possível também que a consciência morfológica esteja associada ao desempenho da escrita em geral. Estudos têm demonstrado que a consciência morfológica contribui para leitura e escrita em diferentes ortografias alfabéticas. Colé, Marec-Breton, Royer e Gombert; (2003) e Sénéchal, Basque e Leclaire (2006) demonstraram essa relação estudando crianças falantes do francês. Contudo, o francês é uma língua com muitas 90 irregularidades ortográficas de base morfológica. O português é uma língua interessante para se investigarem a consciência morfológica e sua relação com a leitura e a escrita, por haver palavras regulares em termos das correspondências entre letra e som e, ao mesmo tempo, uma rica estrutura morfológica. Alguns estudos recentes demonstram essa relação para a leitura (Mota, 2011; Mota et al., 2009).

Estudos que investigam a relação entre a consciência morfológica e a ortografia de palavras morfologicamente complexas no português têm sido realizados. Mota (1996) mostrou que, a partir da segunda série, as crianças são capazes de utilizar regras gramaticais para decidir a grafia de palavras flexionadas. Mota e Silva (2007) observaram que as crianças de primeira série com os melhores escores nos testes de consciência morfológica - analogia gramatical (Nunes, Bidman \& Bryant, 1997) e morfologia produtiva (Berko, 1958) - eram também as crianças que escreviam melhor pseudopalavras com ortografia ambígua (ex:"muge"-"mugidor").

Estudando a relação entre ortografia e morfossintaxe, Rego e Buarque (1997) mostraram que a consciência morfossintática contribuiu para o desempenho das crianças na escrita de palavras morfologicamente complexas. Outro estudo que investigou a relação entre consciência morfossintática e desempenho ortográfico de crianças de $2^{\mathrm{a}}$ e $4^{\mathrm{a}}$ séries do Ensino Fundamental (3 e $5^{\circ}$ ano no sistema atual) foi o de Queiroga, Lins, e Pereira (2006). Os resultados desse estudo mostraram uma evolução entre as séries na escrita de palavras e pseudopalavras e na explicitação do conhecimento morfossintático. Encontrou-se também um efeito facilitador do conhecimento morfossintático para a escrita.

Uma fraqueza dos estudos feitos em ortografias regulares, como o português, reside no fato de que se pesquisa a relação entre a consciência morfológica e a escrita de palavras cuja grafia depende de regras morfológicas (ex: "laranja" - "laranjeira"). Precisamos explorar mais o papel dessa habilidade, pois há ainda poucos estudos na área. Uma das questões a serem levantadas é se essa habilidade está associada à escrita em geral ou só apenas à escrita de palavras morfologicamente complexas.

A questão da relação entre a consciência morfológica e a escrita é ainda mais complexa do que simplesmente estabelecer em quais níveis essa habilidade afeta a escrita. A consciência morfológica é parte de uma 
habilidade mais geral chamada habilidade metalinguística, que está associada ao desenvolvimento linguístico. Precisamos estabelecer se quando se observa uma contribuição da consciência morfológica para escrita, está se observando uma contribuição específica da consciência morfológica. Nunes e Bryant (2006) mostram que o desenvolvimento do vocabulário das crianças está associado ao desenvolvimento da consciência morfológica. Além do vocabulário, a memória de dígitos é uma medida de processamento fonológico e está associada ao desempenho de várias tarefas cognitivas. Na escrita, o processamento fonológico tem um papel importante e já bem documentado (A. Capovilla \& F. Capovilla, 2009). É preciso verificar se a contribuição da consciência morfológica se sustenta mesmo quando se controla o efeito da variância partilhada com essas outras habilidades metalinguísticas. Pode ser que a contribuição na verdade seja devida as outras habilidades metalinguísticas correlacionadas a ela.

Este estudo explorou a relação entre consciência morfológica e o desempenho da escrita por meio de correlações. É um fato conhecido, entretanto, que correlações não estabelecem relações de causa e efeito, nem controlam o efeito de variáveis estranhas. Parte da associação entre a consciência morfológica e a escrita pode ser influenciada pela variância partilhada por essa habilidade e a habilidade de vocabulário e de memória. Quanto melhor o vocabulário da criança, melhor pode ser sua habilidade de refletir sobre os morfemas e sua habilidade de escrita. Correlações parciais podem ajudar a isolar esses fatores e a mostrar se a consciência morfológica ainda continua a contribuir para a escrita.

\section{Método}

\section{Participantes}

A amostra constitui-se de 52 crianças, sendo 25 do $2^{\circ}$ ano e 27 do $3^{\circ}$ ano do Ensino Fundamental de uma escola pública federal situada na região urbana de Juiz de Fora (MG). A média de idade das crianças do 20 ano era de 95,9 meses (DP=3,85) e a do 30 ano, 106,6 meses $(D P=3,94)$. A participação no estudo dependeu da assinatura, pelo responsável, do termo de consentimento livre e esclarecido.

\section{Instrumentos}

A) Tarefa de analogia gramatical (Nunes, Bindman \& Bryant, 1997): nessa tarefa, a criança tinha que produzir uma palavra morfologicamente complexa a partir de uma palavra-alvo, aplicando a mesma relação de derivação de um par previamente dado, como, por exemplo: "pedra-pedreiro; leite- ?" e assim sucessivamente com os outros pares. Foram criados dez itens.

B) Tarefas de decisão morfológica - Raiz: nessa tarefa a criança tinha que decidir qual palavra era da mesma família que a palavra-alvo. As duplas de palavras foram pareadas pelo número de letras e a frequência de ocorrência na escrita (Pinheiro, 1996). Como não há índices de familiaridade para o português, as palavras foram pareadas por frequência para garantir minimamente um equilíbrio na familiaridade entre elas. A lista de exercícios consistia de dez grupos de três palavras envolvendo prefixos e dez grupos de palavra envolvendo sufixos.

C) Teste de escrita - Teste de Desempenho Escolar - TDE (Stein, 1994): os itens de leitura de palavras isoladas e escrita do TDE foram aplicados para avaliar o desempenho na leitura e escrita das crianças. Por meio desse instrumento, que apresenta propriedades psicométricas satisfatórias, incluindo evidências de validade de critério e bom índice de consistência interna $(\alpha>0,70)$, foi avaliado o desempenho na leitura e escrita das crianças. Este estudo faz parte de um projeto maior que investiga o processamento morfológico e a escrita no português. Outro ditado de palavras isoladas foi aplicado, mas os dados desse ditado e da leitura não serão discutidos nesse artigo.

D) Escala de inteligência Wechsler para criançasWISC III (Weschler, 1991): os subtestes de vocabulário, compreensão e dígitos foram aplicados, e foram utilizados os escores ponderados. Esse teste foi aplicado para se controlar a influência do desenvolvimento cognitivo na aquisição da leitura e da escrita. Os coeficientes de fidedignidade para esses subtestes foram calculados pelo método de Guttmam e mostram que apresentam bons índices de consistência interna (coeficientes maiores que 0,60).

\section{Procedimentos}

As crianças foram avaliadas individualmente em duas sessões de 30 a 40 minutos: na primeira, foram 
realizados os testes de analogia gramatical, e dois subtestes do WISC III (vocabulário e dígitos); na segunda sessão, foram aplicados o ditado de palavras, as tarefas de decisão morfológica, e dois subtestes do TDE. Este estudo concentra apenas a análise dos resultados no TDE - Escrita.

Nas tarefas de decisão morfológica - Raiz, era dada a seguinte instrução: "algumas palavrinhas são da mesma família do que outras. Por exemplo, a palavra 'conta' e a palavra 'reconta' são da mesma família. Já a palavra 'bola' e 'rebola` não são da mesma família. Eu vou falar para você uma palavra e depois vou falar mais outras duas e você vai me dizer qual das duas é da mesma família da primeira". Por fim, realizava-se um exemplo com a criança: "a palavra 'gela' é da mesma família que "congela" ou "conversa"? Caso a criança errasse, explicava-se a forma correta e, se acertasse, iniciava-se a tarefa. Depois do exemplo, iniciava-se a aplicação da tarefa, mesmo que a criança não conseguisse acertá-lo.

Na tarefa de analogia gramatical (Nunes, Bindman \& Bryant, 1997), com a instrução de que muitas palavras poderiam ser relacionadas, a aplicadora apresentava um par de palavras relacionadas e pedia à criança que ouvisse uma palavra e criasse outra relacionada, como no exemplo. A tarefa era iniciada sempre pelo exemplo:"pedra-pedreiro; leite-?"e assim, sucessivamente, eram pronunciadas as demais palavras-alvo.

Os dois subtestes do WISC III (vocabulário e dígitos) e os dois subtestes do TDE foram aplicados segundo o procedimento padrão de aplicação do manual.

\section{Resultado}

Este estudo se propôs a investigar a relação entre a consciência morfológica e o desenvolvimento da escrita medida pelo TDE. Para isso, os resultados foram analisados em duas etapas: na primeira etapa, exploramos a relação entre as tarefas de consciência morfológica e os escores no TDE. Na segunda etapa, exploramos a especificidade dessa relação, sendo os resultados obtidos nos subtestes do WISC III utilizados como forma de controle da influência do desenvolvimento cognitivo 92 na aquisição da escrita.

\section{Correlações entre o TDE - Escrita e as medidas de consciência morfológica}

Entre as tarefas de consciência morfológica, apenas as tarefas de analogia gramatical correlacionaram-se de forma moderada significativa e positiva com o TDE $(r=0,49, p<0,01)$, que também apresentou correlações fortes significativas com os escores na tarefa de dígitos $(r=0,63, p<0,01)$ e fracas com os escores no subteste de vocabulário $(r=0,37, p<0,01)$. Nenhuma outra medida correlacionou-se de forma significativa com a escrita (Tabela 1).

A memória auditiva é uma medida de processamento fonológico, que se correlacionou tanto com o escore do TDE quanto com a tarefa de analogia gramatical (consciência morfológica). É possível que a consciência morfológica seja produto de uma habilidade metalinguística mais geral resultante do processamento fonológico (Mota, Annibal \& Lima, 2008). Portanto, esses resultados requerem que verifiquemos a especificidade da relação entre consciência morfológica e escrita por meio da aplicação de controles adequados. Nesse caso, correlações parciais controlando para a variância partilhada pela tarefa de dígitos (processamento morfológico) e vocabulário foram computadas. Os resultados mostram uma relação que se mantém significativa depois dos controles terem sido executados $(r=0,29$, $p<0,038)$.

\section{Discussão}

O presente estudo se propôs a investigar a relação entre a consciência morfológica, aspectos cognitivos e a escrita de palavras medida pelo TDE. Os

Tabela 1. Coeficientes de correlação de Pearson para as tarefas de consciência morfológica, vocabulário, dígitos e ditado de palavras - TDE.

\begin{tabular}{|c|c|c|c|c|c|}
\hline & \multicolumn{2}{|c|}{ Decisão } & \multirow{2}{*}{ Vocabulário } & \multirow{2}{*}{ Dígito } & \multirow{2}{*}{$\begin{array}{l}\text { TDE - } \\
\text { escrita }\end{array}$} \\
\hline & Prefixo & Sufixo & & & \\
\hline Analogia & $-0,10$ & 0,01 & $0,33^{*}$ & $0,35^{* *}$ & $0,49^{* *}$ \\
\hline Decisão prefixo & & $0,48^{* *}$ & $-0,02$ & 0,01 & $-0,10$ \\
\hline Decisão sufixo & & & $-0,03$ & 0,10 & 0,12 \\
\hline Vocabulário & & & & 0,18 & $0,37^{* *}$ \\
\hline Dígito & & & & & $0,63^{* *}$ \\
\hline
\end{tabular}

${ }^{*} p<0,05 ;{ }^{* *} p<0,05$. TDE: Teste de Desempenho Escolar. 
resultados mostram que a medida de processamento fonológico, tarefa de dígito, mostrou uma forte associação com a escrita. Esses resultados são congruentes com os de vários estudos que demonstram que o processamento fonológico tem um papel fundamental na aquisição da língua escrita e que esses resultados se repetem para diversas línguas alfabéticas (Barreira \& Maluf, 2003; Bradley \& Bryant, 1983; A. Capovilla \& F. Capovilla; 2000, Cardoso-Martins, 1995; Cuninghan, 1990; Guimarães, 2003; Santos, 1996).

Muitos estudos chamam a atenção para o fato de o processamento fonológico poder ter um papel mais importante na escrita do que na leitura. Nation e Hulme (1997) lembram que, na leitura, todas as pistas gráficas estão presentes, enquanto na escrita, as crianças têm que representar o som das palavras com pouca ou nenhuma ajuda do contexto e dessas pistas. Assim, é possível que o processamento fonológico seja mais importante na escrita do que o processamento sintático-semântico (Rego \& Bryant, 1993). É interessante ressaltar que, no presente estudo, o índice de correlação entre a medida de dígito (processamento fonológico) e a escrita foi mais forte do que com as outras medidas (vocabulário e consciência fonológica).

A relação entre a consciência morfológica e a escrita também foi demonstrada através dos índices de correlação obtidos. As correlações positivas e significativas entre a medida de escrita do TDE e as medidas de analogia gramatical foram moderadas, mas significativas. As crianças que tiveram maiores escores no TDE foram aquelas com maiores escores na tarefa de consciência morfológica.

Correlações parciais mostraram que a relação entre o processamento morfológico e a escrita se mantém mesmo controlando variáveis como memória auditiva e vocabulário. Esse é um dado importante, pois o efeito da consciência morfológica sobre a escrita continua a existir de forma independente, mostrando a importância dessa habilidade, que traz uma contribuição única, ainda que menor do que a do processamento fonológico para escrita, e precisa ser considerada nos modelos cognitivos sobre a escrita.

Assim, de modo geral, os resultados desse estudo sugerem que, além do processamento fonológico, o processamento morfológico contribui para escrita no português. Embora correlações não estabeleçam rela- ções de causa e efeito, esses resultados ressaltam a importância dessa questão e a necessidade desses resultados serem mais bem explorados. Por exemplo, utilizando-se ditados em que houvesse palavras simples e morfologicamente complexas e comparando-se a performance entre eles, poderia se estabelecer se a contribuição da consciência morfológica aumenta com a complexidade ortográfica.

\section{Referências}

Berko, J. (1958). The child's learning of English morphology. Word, 14, 150-177.

Barrera, S., \& Maluf, M. R. (2003). Consciência metalingüística e alfabetização: um estudo com crianças da primeira série do ensino fundamental. Psicologia: Reflexão e Crítica, 16 (3), 491-502.

Bradley, L., \& Bryant, P. (1983). Categorizing sounds and learning to read: a causal connection. Nature, 301 (S899), 419-421.

Capovilla, A., \& Capovilla, F. (2000). Efeitos do treino de consciência fonológica em crianças com baixo nível sócio-econômico. Psicologia: Reflexão e Crítica, 13 (1), 7-24.

Capovilla, A., \& Capovilla, F. (2009). A consciência fonológica e sua importância para a aquisição da linguagem escrita. In M. Mota (Org.), Desenvolvimento metalingüístico: questões contemporâneas (pp.19-40). São Paulo: Casa do Psicólogo.

Cardoso-Martins, C. (1995). Consciência fonológica e alfabetização. Petrópolis: Vozes.

Carlisle, J. (1988). Knowledge of derivational morphology and spelling ability in fourth, six, and eight graders. Applied Psycholinguistics, 9 (3), 247-266.

Carlisle, J. (1995). Morphological awareness and early reading achievement. In L. Feldman (Ed.), Morphological aspects of language processing (pp.189-211). Hillsdale: Lawrence Erlbaum Associates.

Carlisle, J. (1996). An exploratory study of morphological errors in children's written stories. Reading and Writing: An Interdisciplinary Journal, 8(1), 61-72.

Carlisle, J. (2000). Awareness of the structure and meaning of morphologically complex words: impact on reading. Reading and Writing: An Interdisciplinary Journal, 12 (3), 169-190.

Carlisle, J., \& Fleming, J. (2003). Lexical processing of morphologically complex words in the elementary years. Scientific Studies of Reading, 7 (3), 239-253.

Colé, P., Marec-Breton, N., Royer, C., \& Gombert, J. E. (2003). Morphologie des mots et apprentissage de la lecture. Reeducation Orthophonic, 213 (1), 57-60.

Cunningham, A. E. (1990). Implicit versus explicit instruction in phonemic awareness. Journal of Experimental Child Psychology, 50 (3), 429-444. 
Deacon, S., \& Bryant, P. (2005). What young children do and do not know about the spelling of inflections and derivations. Developmental Science, 8 (6), 583-594.

Deacon, S., \& Kirby, J. (2004). Morphological awareness: just "more phonological"? The roles of morphological and phonological awareness in reading development. Applied Psycholinguistics, 25 (2), 223-238.

Duncan, L., Casalis, S., \& Colé, P. (2009). Early metalinguistic awareness of derivational morphology: observations from comparison of English and French. Applied Psycholinguistics, 30 (3), 405-440.

Guimarães, S. (2003). Dificuldades no desenvolvimento da lectoescrita: o papel das habilidades metalinguísticas. Psicologia: Teoria e Pesquisa, 19 (1), 33-45.

Lehtonen, A., \& Bryant, P. (2005). Active players or just passive bystanders? The role of morphemes in spelling development in a transparent orthography. Applied psycholinguistics, 26 (2), 137-155.

Mann, V. (2000). Introduction to special issue on morphology and the acquisition of alphabetic writing systems. Reading and Writing: an Interdisciplinary Journal, 12 (3), 143-147.

Mota, M. (1996). Children's role of grammatical rules in spelling. Unpublished doctoral dissertation, University of Oxford, United Kingdom.

Mota, M. (2011). Consciência morfológica, aspectos cognitivos da linguagem e reconhecimento de palavras. Interação em Psicologia, 15 (1), 21-26.

Mota, M., Annibal, L., \& Lima, S. (2008). A Morfologia derivacional contribui para a leitura e escrita no português? Psicologia: Reflexão e Crítica, 21 (2), 311-318.

Mota, M., \& Silva, K. (2007). Consciência morfológica e desenvolvimento ortográfico: um estudo exploratório. Psicologia em Pesquisa, 1 (2), 86-92.

Mota, M., Lisboa, R., Dias, J. , Gontijo, R., Mansur-Lisboa, S. F., Silva, D. A., et al. (2009). Relação entre consciência morfológica e leitura contextual medida pelo teste de cloze. Psicologia: Reflexão e Crítica, 22 (2), 223-229.

Nagy, W., Berninger, V., \& Abbot, R. (2006). Contributions of morphology beyond phonology to literacy outcome of upper elementary and middle-school students. Journal of Educational Psychology, 98 (1), 134-147.
Nation, K., \& Hulme, C. (1997). Phonemic segmentation, not onset-Rime segmentation, predicts early reading and spelling skills. Reading Research Quarterly, 32 (2), 154-167.

Nunes, T., Bindman, M., \& Bryant, P. (1997). Morphological strategies: developmental stages and processes. Developmental Psychology, 33 (4), 637-649.

Nunes, T., \& Bryant, P. (2006). Improving literacy by teaching morphemes. London: Routledge.

Pinheiro, A. M. V. (1996). Contagem defrequência de ocorrência de palavras expostas a crianças na faixa pré-escolar e séries iniciais do 10 grau (Software) São Paulo: Associação Brasileira de Dislexia.

Queiroga, B., Lins, M., \& Pereira, M. (2006). Conhecimento morfossintático e ortografia em crianças do ensino fundamental. Psicologia: Teoria e Pesquisa, 22 (1), 95-99.

Rego, L., \& Bryant, P. (1993). The connections between phonological, syntactic and semantic skills and children's reading and spelling. European Journal of psychology, 8 (3), 235-246.

Rego, L., \& Buarque, L. (1997). Consciência sintática, consciência fonológica e aquisição de regras ortográficas. Psicologia: Reflexão e Crítica, 10 (2), 199-217.

Santos, A. A. A. (1996). A influência da consciência fonológica na aquisição da leitura e da escrita. In F. F. Sisto, G. C. Oliveira, L. D. T. Fini, M. T. C. C. Souza \& R. P. Brenelli (Eds.), Atuação psicopedagógica e aprendizagem escolar (pp.213-247). Petrópolis: Vozes.

Sénéchal, M., Basque, M. T., \& Leclaire, T. (2006). Morphological knowledge as revealed in children's spelling accuracy and reports of spelling strategies. Journal of Experimental Child Psychology, 95 (4), 231-254.

Stein, L. M. (1994). TDE: Teste de desempenho escolar: manual para aplicação e interpretação. São Paulo: Casa do Psicólogo.

Wechsler, D. (1991) WISC-III: Escala de inteligência Weschsler para crianças. São Paulo: Casa do Psicólogo.

Recebido em: 22/6/2009

Versão final reapresentada em: 17/10/2011

Aprovado em: 19/10/2011 